\title{
Hospital-Based Surveillance: An Effective Tool for Infectious Disease Control
}

\author{
Shefalee tai Vernekar', Hemangini Shah² \\ ${ }^{1,2}$ Department of Preventive and Social Medicine, Goa Medical College. \\ DOI: https://doi.org/10.24321/2454.325X.201810
}

\section{Abstract}

Objectives: (1) To determine the profile of reported infectious diseases. (2) To analyze the reported infectious diseases with reference to certain factors. (3) To evaluate the functionality of the notification system.

Methodology: The hospital record-based study was carried out at a tertiary care hospital in Goa. The "Malaria, Dengue, Encephalitis Notification Reports" for a period of 18 months (Jan 2016-Jun 2017) were scrutinized.

Data was entered in excel and analyzed in SPSS version 22.

Results: A total of 2360 cases were recorded as per reports. 2043 (86.5\%) of these were cases of malaria, 225 (9.6\%) dengue, 55 (2.3\%) encephalitis, 37 (1.6\%) leptospirosis, and zero cases of chikungunya. Among the malaria cases, majority of the cases notified were plasmodium vivax-type. There were a total of 30 deaths reported, majority being due to leptospirosis 7 (23\%) and encephalitis 7 (23\%). The highest case fatality rate of 22 was due to cerebral malaria.

Only 48 out of 346 (14\%) of the reports were duly complete. Incorrect notification was seen in $2 \%$ of the reports. Many a times (25\%), the reports were not sent on a daily basis.

Conclusion: The hospital-based surveillance is an effective notification system for cases reporting to the hospitals. It is a cost-effective method of surveillance. If the problems of inconsistency, incompleteness, untimely reporting, etc., are solved, it has potential to revolutionize by timely detection and control measures on impending disease outbreaks.

Keywords: Surveillance, Hospital-based, Infectious, Disease

\section{Introduction}

In India, the range and burden of infectious diseases is enormous. ${ }^{1}$ Many infectious diseases are preventable/ controllable and it includes collection of accurate assessment of data (such as surveillance data for specific conditions), outbreak detection and investigation, development of appropriate control strategies (both short and long term) based on specific epidemiologic data. ${ }^{2}$ Although the burden of infectious diseases has decreased as a result of overall socioeconomic progress and increased use of vaccines and antimicrobials, India has not yet succeeded in controlling many old, new, or resurgent infectious diseases, ${ }^{1}$ and many of these diseases are epidemic prone. However, precautionary measures can be taken to reduce the risks of outbreaks. ${ }^{3}$

Corresponding Author: Dr. Shefalee Pai Vernekar, Department of Preventive and Social Medicine, Goa Medical College.

E-mail Id: shefa_pv@yahoo.co.in

Orcid Id: https://orcid.org/0000-0002-5487-1878

How to cite this article: Vernekar SP, Shah H. Hospital-Based Surveillance: An Effective Tool for Infectious Disease Control. Int J Preven Curat Comm Med 2018; 4(2): 4-9. 
Surveillance can be defined as 'Ongoing systematic collection, collation, analysis and interpretation of data and the dissemination of information to those who need to know in order that action may be taken' - Information for Action. Effective infectious diseases control relies on an effective surveillance and response system that promotes better coordination and integration of surveillance function. ${ }^{4}$

The surveillance data serves as an early warning system and helps in routine program monitoring. An early warning system serves to:

- Detect outbreaks of diseases in a timely manner

- Inform appropriate and effective public health responses

- Determine the distribution and spread of diseases

- Illustrate the epidemiology of new diseases

- Provide information to categorize the outbreak as of national or international importance

- Provide data to evaluate control measures

The surveillance systems for monitoring effectiveness of control programs serve to estimate disease burden, identify risk groups, determine incidence trends over time, measure outcomes and impacts of preventive and public health interventions, and evaluate the overall control interventions. ${ }^{5}$

Big gaps however exist in the existing surveillance system for infectious diseases, and this needs to be addressed. ${ }^{1}$ Hospitals can play a pivotal role in generating early warning signals of an impending outbreak by sharing this information to the local health authorities. ${ }^{3}$

It is important to identify critical system gaps in the surveillance and response system for infectious diseases for its effective functioning. Hence this study was carried out with the following aims and objectives:

- To determine the profile of reported infectious diseases

- To analyze the reported infectious diseases with

reference to certain factors

- To evaluate the functionality of the notification system

\section{Methodology}

The hospital record-based study was carried out at a tertiary care hospital in Goa where there exists a daily reporting system for diseases such as malaria, dengue, encephalitis, chikungunya and leptospirosis through a report called the "Malaria, Dengue, Encephalitis Notification Report". Information documented in registers by the concerned deputed staff in their designated areas is gathered from all the wards, outpatient departments as well as the laboratories. Data is then compiled and sent on a daily basis to the NVBDCP cell at the Directorate of Health Services.

Data was collected by scrutinizing all the details like name, age, sex, address, admission and discharge in case of inpatients, etc., in the duly filled Malaria, Dengue, Encephalitis Notification Reports maintained in the MRD for a period of 18 months (Jan 2016-Jun 2017) after obtaining permission from Medical Record Department and Institutional Ethics Committee. This daily report also provided data on the total number of cases due to that particular disease as well as death, if any. Data was entered in Excel and analyzed in SPSS version 22.

\section{Results}

Total 346 reports were sent for the period of 18 months fromJanuary 2016 to June 2017. Majority of the cases, i.e., 476 (56\%) were among the age group 19-44 years. Among the reported cases, children less than 5 years comprised $119(14 \%)$, between 6 and 18 years were150(18\%) and above 45 years $100(12 \%)$ of the cases.1737(74\%) of the cases were among males. 851 (60\%) of the cases resided in an urban area, $520(36 \%)$ in rural and the remaining 52 (4\%) were migrants. Majority of the migrants were from the neighboring states of Karnataka $(75 \%)$ and Maharashtra (17\%) (Table 1).

Table 1.Sociodemographic Details of Cases

\begin{tabular}{|c|c|c|}
\hline Variable & Number & Percentage \\
\hline \multicolumn{3}{|c|}{ Age (years): $\mathbf{n = 8 4 5}$} \\
\hline$\leq 5$ & 119 & 14 \\
\hline $6-18$ & 150 & 18 \\
\hline $19-44$ & 476 & 56 \\
\hline$\geq 45$ & 100 & 12 \\
\hline \multicolumn{3}{|c|}{ Sex: $\mathbf{n = 2 3 6 0}$} \\
\hline Male & 1737 & $\mathbf{2 0}$ \\
\hline Female & 623 & 60 \\
\hline Urban & Residence: $\mathbf{n = 1 4 2 3}$ & 36 \\
\hline Rural & 851 & 4 \\
\hline Migrants & 520 & \\
\hline
\end{tabular}


A total of 2360 cases were recorded as per reports. 2043 (86.5\%) of these were cases of malaria, 225 (9.6\%) dengue, 55 $(2.3 \%)$ encephalitis, $37(1.6 \%)$ leptospirosis and zero cases of chikungunya (Table 2 ). Among the malaria cases, majority of the cases notified were plasmodium vivax type, i.e., 1609 (68.1\%) while plasmodium falciparum were 313 (13.1\%) and mixed were 112 (4.7\%). 9 (0.4\%) of the cases reported were due to cerebral malaria (Table 3).

Table 2.Distribution of Cases, Deaths and Case Fatality Rates Based on Type of Infectious Disease

\begin{tabular}{|c|c|c|c|c|}
\hline \multirow{2}{*}{ Type of Infectious Disease } & \multicolumn{2}{|c|}{ Cases } & \multirow{2}{*}{ Deaths } \\
\cline { 2 - 5 } & Number & Percentage & & 23.3 \\
\hline Malaria & 2043 & 86.5 & 10 & 02.7 \\
\hline Dengue & 225 & 9.6 & 6 & 13 \\
\hline Encephalitis & 55 & 2.3 & 7 & 19 \\
\hline Leptospirosis & 37 & 1.6 & 0 & 00 \\
\hline Chikungunya & 0 & 0 & 30 & -- \\
\hline Total & 2360 & 100 & & 7 \\
\hline
\end{tabular}

Table 3.Distribution of Cases, Deaths and Case Fatality Rates Based on Type of Malaria

\begin{tabular}{|c|c|c|c|c|}
\hline \multirow{2}{*}{ Type of Malaria } & \multicolumn{2}{|c|}{ Cases } & \multirow{2}{*}{ Deaths } \\
\cline { 2 - 4 } & Number & Percentage & 5 & 0.3 \\
\hline P Vivax & 1609 & 68.1 & 3 & 1 \\
\hline P Falciparum & 313 & 13.3 & 0 & 0 \\
\hline Mixed & 112 & 4.7 & 2 & 22 \\
\hline Cerebral & 9 & 0.4 & 10 & 23.3 \\
\hline Total & 2043 & 86.5 & & 0 \\
\hline
\end{tabular}

1551 (75\%) of the cases were seen at outpatient level and 809 (35\%) required admission for treatment. There were a total of 30 deaths reported, majority being due to leptospirosis 7 (23\%) and encephalitis 7 (23\%) (Table 2). The case fatality rates due to individual diseases were also calculated based on the data as shown in Table 2 . The highest case fatality rate of 22 was due to cerebral malaria (Table 3 ).

A month-wise distribution of the cases depicted seasonal variation. Most of the cases were in the monsoon season with a peak in the month of July (Fig. 1).

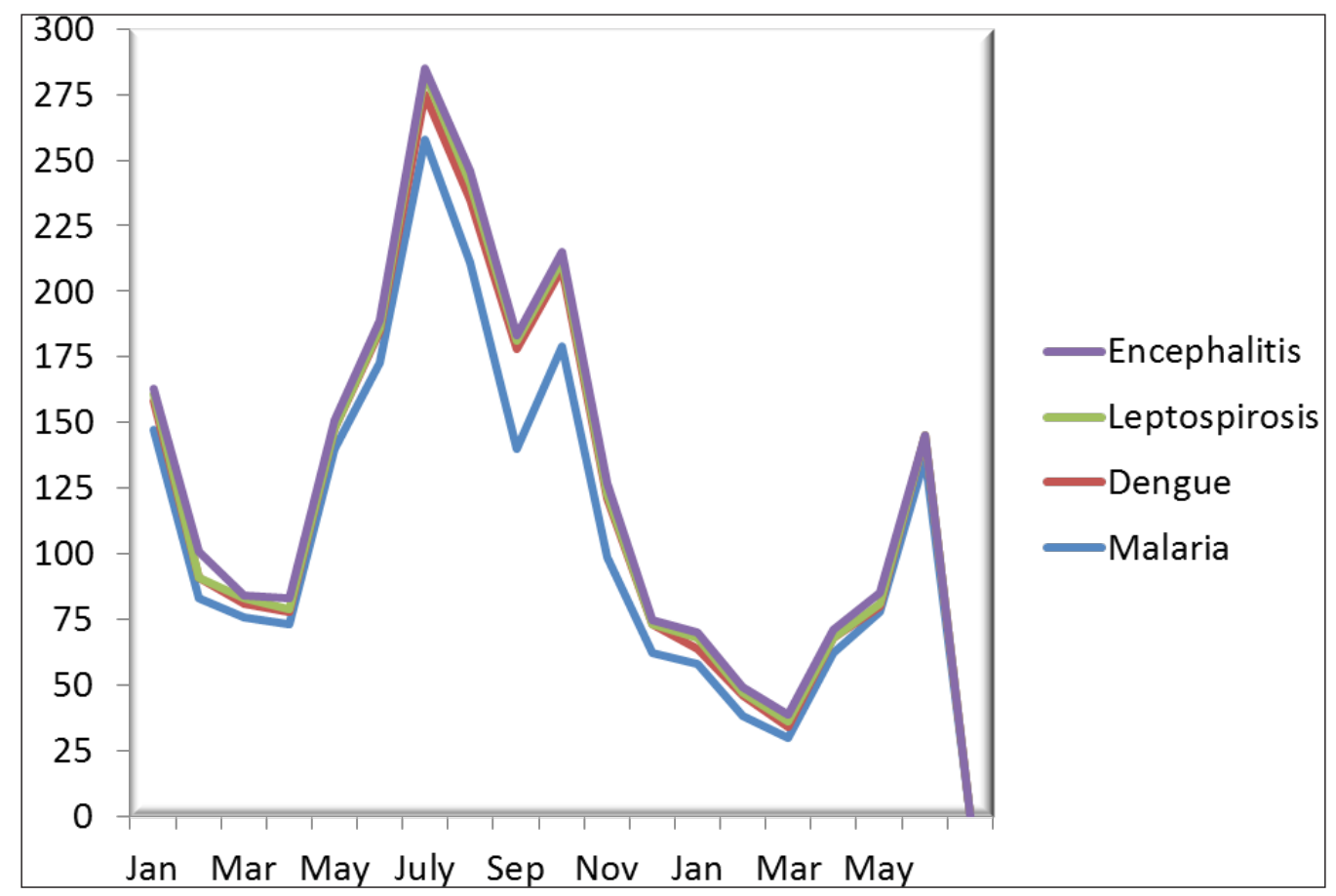

Figure 1.Month-Wise Distribution of Cases 


\section{Evaluation of Notification System under the NVBDCP}

The notification system under NVBDCP, followed in the tertiary care hospital, Goa, was evaluated with respect to the following points:

\section{Structure}

Information about OPD patients is maintained by the laboratory assistants as the patients come for blood and other investigations in the laboratories. In the wards, staff nurses maintain the registers. This information is collected by the clerk from the Medical Records Department (MRD) on a daily basis. This data is then compiled and checked by the nodal officer incharge, from the Department of Preventive and Social Medicine (PSM) and the reports thus generated are sent to the National Vector Borne Disease Control Program (NVBDCP) cell of the Directorate of Health Services (DHS) electronically through the Office of the Medical Superintendent of the hospital. Copies of the reports are maintained at the MRD, Department of PSM, and the office of the Medical Superintendent (Fig. 2).
OPD. 25 out of $346(7.2 \%)$ had list of cases without the names of the patients.

This was sometimes due to error in noting down the details from the registers, and also because the registers maintained by wards, OPDs and laboratories, were also not updated fully in some cases, particularly the address and contact numbers of the patients, and sometimes the error was in noting down the details from the registers.

\section{Incorrect notification}

$2 \%$ of the reports were incorrect, wherein the data of the number of cases did not match with the list of patients' names. This could have been either due to unavailable patient details or wrong number of cases noted down while filling the form.

\section{Timeliness}

Many a times (25\%), the reports were not sent on a daily basis. There was a delay of 1 or more days but at one particular time there was a delay of 6 days. This happens

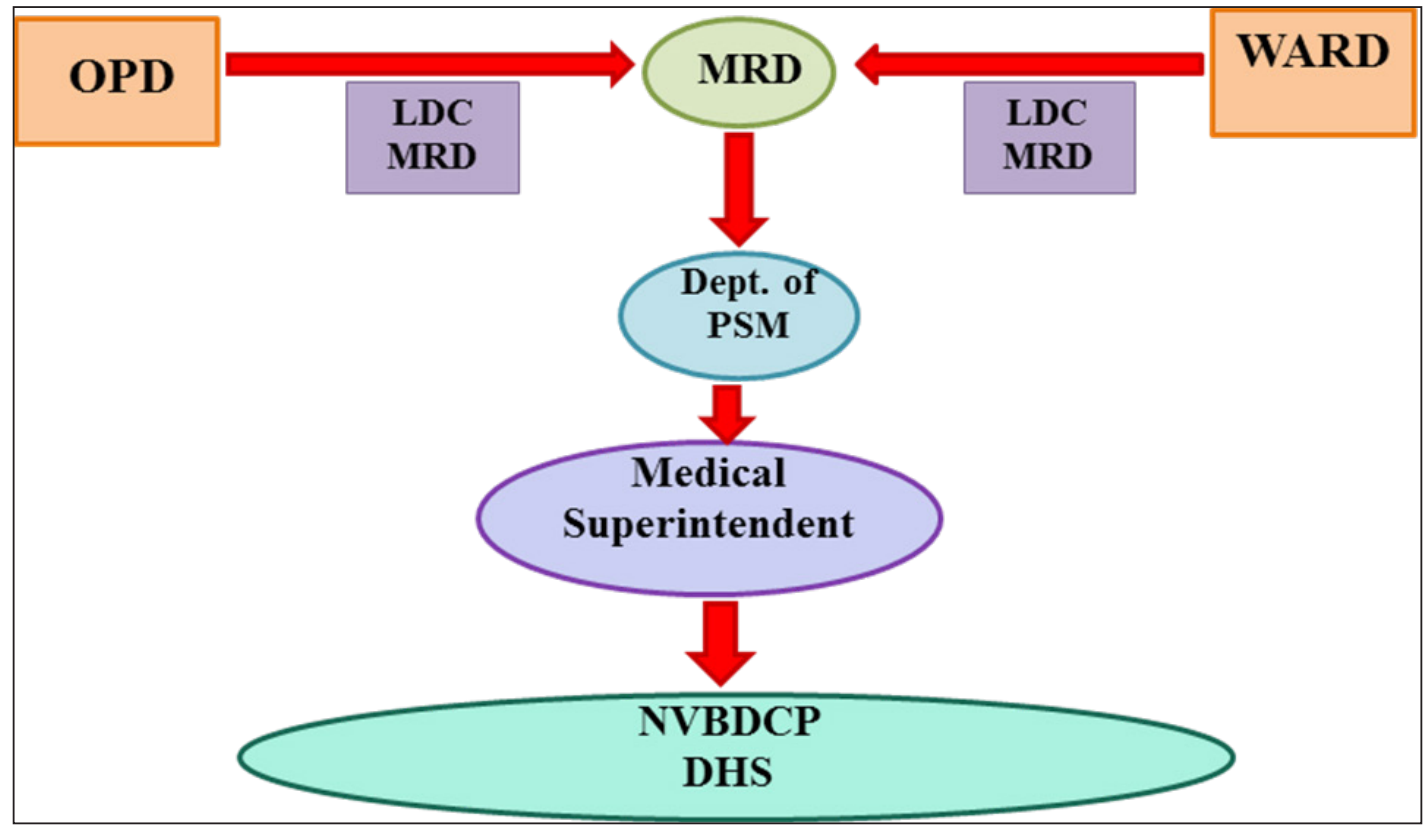

Figure 2.Structure of the Notification System

\section{Completeness}

The individual reports were checked for completeness in the form of all details filled in the reports as per the format. It was found that none of the reports were fully complete. Only 48 out of $346(14 \%)$ of the reports were duly complete with data about the address, contact numbers and age of the patients. The ward of admission was mentioned in all the reports, wherever applicable. However, the date of admission was present only in 300 (86.7\%). In case of OPD patients, the name of the OPD was not mentioned. All the patients were mentioned under one category as due to other work commitments in the MRD, particularly when there is excess workload or other staff is on leave.

\section{Usefulness}

The information obtained from the reports was the following:

- Number of Malaria (vivax/falciparum/mixed/cerebral)/ dengue/leptospirosis/encephalitis/chikungunya cases

- Name/age/sex/address/contact numbers

- Ward of admission/date of admission

- Death, if any 
This information, sent to the DHS, is utilized for further action at the community level. The concerned primary medical officer is notified about the details of the report by the DHS personnel, especially the information about the cases detected in the area under the PHC. Also active search for cases is initiated, vector-breeding sites are identified, and control measures are taken. Awareness activities are also carried out.

The data about name, residence of patients, and contact numbers collected from reports helped in tracing of patients, carrying out active search and vector management in the area of residence. The age and sex of the patients is also important from epidemiological point of view.

\section{Discussion}

This study shows that the notification system helps to collect data on the number of cases, fatalities due to the diseases as well as sociodemographic details. Also, seasonal variations and trends in the above diseases can be identified. However, certain weaknesses do exist in the system.

The public health surveillance system should operate in a manner that allows effective dissemination of health data so that decision makers at all levels can readily understand the implications of the information.

The overall structure of the notification system was appropriate. However, the medical and laboratory staff as well as the data collector (clerk from the MRD) were not aware of the importance and public health implications of correct, complete and timely reporting.

Incomplete patient details, incorrect reports, and delays were major weaknesses that exist in this notification system. In our study, we found that none of the reports was complete in all aspects. Data missing was mainly the sociodemographic details, i.e., age and addresses of the patients, which are crucial for epidemiological information for disease surveillance. The entry about provisional diagnosis in these maintained registers is sometimes either not complete or not even legible. Timeliness and completeness of reports reflect the effectiveness of data collection and transmission and are proxy indicators of the alertness of the surveillance system. An alert system will have timeliness and completeness approaching $100 \%$. Completeness of reporting units also gives one an idea about the reliability of the data as stated by Srivastava et al. ${ }^{6}$ The inclusion of socioeconomic variables into a surveillance system, which are often missing in many existing surveillance studies, is a challenging aspect. ${ }^{7}$ Data not linked to socioeconomic information does not facilitate the assessment of health disparities. These indicators thus help to identify non-functional or poorly functioning reporting system.
The primary purpose of reporting specific infectious diseases is to trigger an appropriate public health response so that further illness can be prevented. Bean et al. states that electronic data transmission is likely to be more timely than conventional paper-based systems. ${ }^{8}$ Lack of medical records, especially in electronic format, is a major gap. CDC recommends usage of mobile phones/IT for regular transmission of reports and timely reporting. ${ }^{9}$

The major benefit of the notification system is the transfer of information from the tertiary care hospital to the directorate of health services. This enables notification of concerned medical officers and health workers to conduct epidemiological or etiological investigations on many of the reported diseases and necessary interventions can be taken.

\section{Conclusion}

The hospital-based surveillance is an effective notification system for cases reporting to the hospitals. It is a costeffective method of surveillance. Though there are problems of inconsistency, incompleteness and untimely reporting, its implementation can be improved. It hints towards need for raising awareness and understanding among the health professionals engaged in medical care about the scope and significance of accurately generating, documenting, compiling and reporting data on health events or diseases. Regular follow up to maintain the sensitivity and specificity of system and proper utilization of available human resources is needed.

Electronic medical records (EMRs), hospital management information systems and; geographical information system (GIS) are possible solutions for the problem of ineligible or missing information in the inpatient and outpatient registers in the hospital.

Hospital-based surveillance in the tertiary care hospital Goa has significantly uncovered the infectious disease burden, improved case and outbreak detection and provided a scope for epidemiology. With its expansion and linkages with other surveillance systems, it has potential to revolutionize by timely detection and control measure on impending disease outbreaks.

\section{Conflict of Interest: None}

\section{References}

1. John TJ, Dandona L, Sharma VP et al. Continuing challenge of infectious diseases in India. Lancet 2011; 377(9761): 252-69.

2. Gorji MAH. A study on national notifiable infectious disease surveillance in India: A managerial perspective. Aligarh Muslim University 2009; 41.

3. National Centre for Disease Control. Training Manual for Medical Officers for Hospital Based Disease 
Surveillance. IDSP 2004;32.

4. WHO African Region : Ethiopia Integrated Disease Surveillance and Response. WHO 2018. Available at:http://www.who.int/countries/eth/areas/ surveillance/en/.

5. World Health Organization. Communicable disease surveillance and response systems - Guide to monitoring and evaluating. epidemic and pandemic alert and response. WHO 2006; 90.

6. Srivastava D, Venkatesh S, Pandey S et al. Completeness and timeliness of reporting under integrated disease surveillance project (IDSP) in rural surveillance unit of Nainital district of Uttrakhand, India. Indian J Prev Soc Med. 2009; 40(3):138-42.

7. Alwan A, MacLean DR, Riley LM et al. Monitoring and surveillance of chronic non-communicable diseases:
Progress and capacity in high-burden countries. Lancet 2010: 376: 1861-68.

8. Bean N, Martin S. Implementing a network for electronic surveillance reporting from public health reference laboratories: An international perspective. Emerg Infect Dis. 2001 Sep-Oct; 7(5): 773-79. Available at: http://www.cdc.gov/ncidod/eid/vol7no5/bean. htm.

9. Centers for Disease Control and Infection. Performance Criteria for Public Health Disease Reporting Systems Operating Twenty-four Hours per Day, Seven Days per Week (24/7); 2003. Available at: http://www.cdc.gov/ epo/dphsi/8city.htm.

Date of Submission: 2018-03-12

Date of Acceptance: 2018-03-16 